\title{
Intrinsic gemcitabine resistance in a novel pancreatic cancer cell line is associated with cancer stem cell-like phenotype
}

\author{
GANG HU ${ }^{1,2^{*}}, \mathrm{FU} \mathrm{LI}^{1 *}, \mathrm{KEDONG}^{*} \mathrm{OUYANG}^{2}, \mathrm{FUBO} \mathrm{XIE}^{2}, \mathrm{XUZHEN} \mathrm{TANG}^{2}, \mathrm{KE} \mathrm{WANG}^{2}$, SUFANG HAN $^{2}$, \\ ZHENZHOU JIANG $^{1}$, MINGHUA ZHU ${ }^{3}$, DANYI WEN ${ }^{2}$, XIAORAN QIN ${ }^{2}$ and LUYONG ZHANG ${ }^{1}$ \\ ${ }^{1}$ National Nanjing Center for Drug Screening, China Pharmaceutical University, Jiangsu 210009; ${ }^{2}$ Shanghai ChemPartner Co., \\ Ltd., Shanghai 201203; ${ }^{3}$ Department of Pathology, Shanghai Changhai Hospital, Shanghai 200433, P.R. China
}

Received August 19, 2011; Accepted October 6, 2011

DOI: $10.3892 /$ ijo.2011.1254

\begin{abstract}
Pancreatic ductal adenocarcinoma (PDA) remains one of the most lethal malignancies in the world, often diagnosed at an advanced stage, resistant to conventional chemotherapy and having high invasive and metastatic potential. The mechanism of drug resistance of PDA is still not clear. In the present study, we established two novel pancreatic cancer cell lines PAXC-002 and PAXC-003 from human primary xenograft models. The cell lines were characterized by morphology, karyotype, pancreatic cancer marker and short tandem repeat (STR) analysis, and growth kinetics and tumorigenicity. The in vitro anti-proliferation test revealed that PAXC-002 cell was intrinsically resistant to the standard of care chemotherapy-gemcitabine, compared with that of PAXC-003 and other widely used pancreatic cancer cell lines. Interestingly, the gemcitabine resistant PAXC-002 cell line was more potent in forming colonies in 3-Dimensional matrigel culture conditions and had a higher percentage of CD133 positive cells, which is recognized as a cancer stem cell marker, compared to the gemcitabine-sensitive PAXC-003 cell line. In this study, we present two novel pancreatic cancer cell lines which could be used for gemcitabine resistance investigation, mechanism identification of pancreatic cancer and anticancer drug screening. The preliminary data indicate that the drug resistance of pancreatic carcinoma cells is associated with a cancer stem cell-like phenotype.
\end{abstract}

Correspondence to: Dr Luyong Zhang, National Nanjing Center for Drug Screening, China Pharmaceutical University, No. 24 Tongjia Xiang, Nanjing, Jiangsu Province 210009, P.R. China

E-mail: lyzhang@cpu.edu.cn

Dr Xiaoran Qin, Shanghai ChemPartner Co., Ltd., No. 5 Building, 998 Halei Road, Zhangjing Hi-Tech Park Pudong New Area, Shanghai 201203, P.R. China

E-mail: xiaoran_qin@yahoo.com

*Contributed equally

Key words: pancreatic ductal adenocarcinoma, resistance, cancer stem cell, gemcitabine

\section{Introduction}

Pancreatic ductal adenocarcinoma (PDA) is one of the most intractable human malignancies, which has an extremely poor prognosis (a 5-year overall survival of $<5 \%$ ). It is the fourth most common cause of cancer death yearly in the United States. Furthermore, the incidence of pancreatic cancer in China increased about four-fold during the past 20 years, and now it is the ninth cancer death cause (2.62 died in 100,000 people in 2005) (1).

Despite the huge effort on research and development of chemotherapies for pancreatic cancer, these are only a few treatment options approved in clinic. Gemcitabine, a novel pyrimidine nucleoside analogue, has become the standardof-care therapy used in patients with pancreatic cancer, which showed advantage over the previously used 5-fluorouracil $(2,3)$. However, no more than $25 \%$ of patients benefit from this therapy, and the palliative treatment with gemcitabine could only prolong patient survival from maximum 4 months to about 6-7 months, with improved life quality $(4,5)$. Moreover, a substantial number of potential drug combinations have been tested clinically, but no convincing results have been obtained (6-9). The drug resistance of pancreatic cancers is still under wide investigation.

There are many different mechanisms involved in gemcitabine resistance. The drug transporters and metabolic enzymes of gemcitabine were extensively studied (10). The expression of human equilibrative nucleoside transporter-1 (hENT-1), which plays a key role in gemcitabine intracellular uptake, was found decreased in the acquired resistant cell line (11), and hENT expression could significantly influence the clinical survival $(12,13)$. The downstream converting enzymes, such as deoxycytidine kinase (dCK), the ribonucleotide reductase M1 (RRM1) and M2 (RRM2) were also found associated with the acquired gemcitabine resistance (14). In addition, the apoptosis-regulation proteins, such as Bcl-2, Bax and Bak were deregulated in pancreatic cancers (15). The multidrug resistance-associated protein (MRP) (16), focal adhesion kinase (FAK) phosphorylation (17), and others were also found involved in drug resistance. However, these studies were mainly conducted on the acquired resistant cell lines, and the intrinsically resistant cell models are rare. 
The cancer stem cells (CSC) have advanced the research of pancreatic cancer resistance. Several groups identified in 2007 the pancreatic cancer stem cells with CD24, CD44, epithelial specific antigen (ESA) triple positive markers or CD133 positive marker, which had increased tumorigenic potential and metastatic activity compared with non-CSC bulk tumor cells $(18,19)$. Moreover, Hermann et al also found that the pancreatic cancer stem cells were resistant to the gemcitabine induced apoptosis (18). Later, Shah et al and Du et al established the acquired gemcitabine-resistant pancreatic cancer cell lines and they found the resistant cells had more cancer stem cell-like phenotypes compared with their parental cells $(20,21)$. Recently, new insights were presented indicating that the resistance of pancreatic cancer was partly due to the pancreatic desmoplastic stroma and poor vascularization in mouse transgenic models, which impaired the drug delivery in vivo (22-24).

In the present study, we established two novel human pancreatic cancer cells lines PAXC-002 and PAXC-003, and both cell lines were well characterized. These low passage tumor cells maintained their clinical and pathological characteristics, which facilitated the translation of clinical knowledge. PAXC-002 cell line was intrinsically resistant to gemcitabine compared with PAXC-003 and other widely used pancreatic cancer cell lines. Furthermore, the gemcitabine resistant PAXC002 cells had more in vitro colony formation ability and CD133 positive cancer stem-like cells. These results indicated that the pancreatic cancer cell resistance may associate with cancer stem cell phenotype. The two cell lines will be useful for pancreatic cancer research and anticancer drug screening.

\section{Materials and methods}

Patient tumors. Human pancreatic ductal adenocarcinoma (PDA) samples were obtained from Shanghai Changhai Hospital in accordance with protocols approved by the Institutional Ethics Committee. The tumor samples were placed in pre-cooled sterile 'collecting saline' (HBSS containing antibiotic-antimycotic) (Invitrogen, USA) and transported on ice to specific pathogenfree (SPF) animal facility of Shanghai ChemPartner Co. Ltd. for human primary tumor establishment, which is accredited by AAALAC.

Generation of human primary xenografts. Six to eight weeksold female SCID mice (Beijing Vital River, China) were used for implantation of patient tumor fragments. The mice were bred in SPF animal facilities for at least three days before experiments. Their care, housing and experiments were in accordance with ChemPartner's IACUC guidelines. The tumor samples were washed twice with fresh pre-cooled collecting saline and then cut with a sterile scalpel blade into $2 \times 2 \mathrm{~mm}$ pieces on ice. Two to three tumor pieces were implanted subcutaneously (s.c.) into the right flank of the mice. The mice were monitored for tumor growth and body weight for up to 10 weeks. The tumor length (L) and width (W) were measured by digital caliper and the tumor volume (TV) was calculated by the following formula: $\mathrm{TV}=1 / 2 \times \mathrm{L} \mathrm{x} \mathrm{W}^{2}$.

Establishment of cell lines. When the tumors grew to 500-700 $\mathrm{mm}^{3}$, the mice were euthanized and the tumors were removed in sterile condition and were used for in vitro primary culture.
The tumors were washed twice with sterile collecting saline, and the necrosis and connective tissue were carefully removed. The tumor tissue was minced finely using sterile scalpel blade and, after extensively washing with culture medium, transferred into a T-25 culture flasks and incubated at $37^{\circ} \mathrm{C}, 100 \%$ humidity with $5 \% \mathrm{CO}_{2}$. The culture medium was RPMI-1640 medium supplemented with $10 \%$ heat-inactived fetal bovine serum (FBS) (Invitrogen), $10 \mu \mathrm{g} / \mathrm{ml}$ human recombinated insulin (Invitrogen) and 1\% antibiotic-antimycotic (Invitrogen). Controlled trypsinizations were done to preferentially remove the contaminating fibroblasts. The cultures were fed twice a week and subcultured when the cells grew to $70-80 \%$ confluence. The mycoplasma contamination was analyzed by PCR detection (MycoScan ${ }^{\mathrm{TM}}$ Mycoplasma Detection Kit, HDB Biosciences, China). The exponentially growing cells were used for future experiments after passage 20. These cell lines were stocked at China Center for Typical Culture Collection (CCTCC).

Cell culture. AsPC-1, BxPC-3, MIA PaCa-2, PANC-1 and Capan-1 cell lines were obtained from ATCC and maintained according to the instruction. AsPC-1 and BxPC-3 cells were maintained in RPMI-1640 + 10\% FBS, Capan-1 cells were cultured in IMDM (Invitrogen) $+20 \%$ FBS, PANC-1 cells were cultured in DMEM (Invitrogen) + 10\% FBS, MIA PaCa-2 cells were cultured in DMEM $+10 \% \mathrm{FBS}+2.5 \%$ horse serum (Invitrogen).

Morphologic analysis. Exponentially growing cells were observed via inverted phase-contrast microscope (Olympus, Japan). Digital pictures were taken from a camera mounted to a microscope (Olympus). Tumor cells were harvested and cultured as a monolayer on sterile chamber slides. Then they were fixed with $4 \%$ formaldehyde in phosphate-buffered saline (PBS), permeabilized in PBS containing 0.1\% Triton $\mathrm{X}-100$ (Sigma, USA), incubated in $0.3 \% \mathrm{H}_{2} \mathrm{O}_{2}$ solution to quench endogenous peroxidase activity, and blocked with $4 \%$ goat serum (Invitrogen) in PBS. Fixed cells were incubated with anti-cytokeratin (Santa Cruz), anti-CA19-9 (Santa Cruz) and anti-CEA (Santa Cruz) antibodies. Then the slides were developed by DAB methods (Maxim, China) and mounted. The slides were observed and visualized by Eclipse Motorized Advanced Research Microscope (Nikon, Japan).

Chromosome analysis. Exponentially growing cells were seeded in new T-75 flasks. When the cells grew to 50-60\% confluence, the cultures were fed with fresh medium containing $0.2 \mu \mathrm{g} / \mathrm{ml}$ colchicines (Sigma) and incubate for another 4-6 h. The $\mathrm{M}$ phase cells were harvest by gently taping and resuspended carefully in pre-warmed $0.075 \mathrm{~mol} / 1 \mathrm{KCl}$ hypotonic solution and then incubate at $37^{\circ} \mathrm{C}$ for $10 \mathrm{~min}$. The cells were fixed in the fix solution (methanol:glacial acetic acid $=3: 1$, freshly prepared) several times. Then the cells were spread on the pre-cooled slide evenly and stained by Giemsa solution (Invitrogen). The chromosome numbers were counted under the microscope (Nikon, Japan) and the chromosome frequency of each cell line was analyzed by Origin software.

STR profiling. Tumor cells were harvested and washed in sterile PBS solution. The genomic DNA was extracted by 
AxyPrep Multisource Genomic DNA Miniprep Kit (Axygen, USA) and the STR repeats were analyzed by AmpF/STR ${ }^{\circledR}$ Identifiler ${ }^{\circledR}$ PCR Amplification Kit (ABI, USA). All manipulations followed the vendors' instructions.

Population doubling time. Tumor cells were trypsinized and inoculated into the 96-well plates (poly-D-lysine coated, blank wall and clear bottoms) (Becton-Dickinson, USA) at the density of 2000 cells/well. The plates were fixed in Prefer Fix solution (Anatech, USA) at different time-points (12, 24, 36, 48, 72 and $96 \mathrm{~h}$ after inoculation). Then the plates were washed with PBS solution, permeabilized in $0.1 \%$ Triton X-100 solution, and stained with $1.5 \mu \mathrm{g} / \mathrm{ml}$ propidium iodide and $100 \mu \mathrm{g} / \mathrm{ml}$ RNase solution. The plates were analyzed by Acumen eX3 instrument (TTP, UK) and the cell numbers in each well were counted. The cell population doubling times (DT) were linearly fitted and calculated by following formula: DT $=\log 2 / \mathrm{S}$ ( $\mathrm{S}$ means the slope of the linear fitted curve of the log cell growth).

In vivo tumorigenicity. Six to eight week-old female SCID mice (Beijing Vital River, China) were bred in the SPF animal facilities and used for the xenograft tumor generation. Tumor cells were expanded and harvested, washed in pre-cooled serumfree 1640 medium and the cell concentration were adjusted to $5.0 \times 10^{7} / \mathrm{ml}$ and placed on ice. The cell suspension was mixed with the matrigel (Becton-Dickinson) at a ratio of 1:1. The cell mixture was inoculated at the right flank of the mice s.c. (5.0x $10^{6}$ cells/mouse, 10 mice for each line). The tumor growth and mouse body weights were monitored twice a week. When the tumor grew to $\sim 1500 \mathrm{~mm}^{3}$, the mice were euthanized and the tumors were collected. The viable tumor tissues were fixed in $4 \%$ formaldehyde, paraffin-embedded and diagnosed (H\&E staining).

In vitro anti-proliferation test. Tumor cells were seeded into 96-well tissue culture plates (Corning, USA) (the optimal cell density were determined by cell growth curve study and the control cells were still exponentially growing at the assay endpoint) in $150 \mu \mathrm{l}$ culture medium. Gemcitabine (Chemiceutical, USA) was added into triplicate wells at the time of cell inoculation (gemcitabine was dissolved in DMSO, started from $100 \mu \mathrm{M}, 1: 5$ serially diluted, 10 points, and the final DMSO concentration in medium was $0.5 \%$ ). After a 96-h incubation, the cell viability in treated and control wells were measured by CellTiter Glo method (Promega, USA) according to the manufacturer's instructions. Drug effects were presented as $\mathrm{IC}_{50}$ drug concentration and inhibition index $\left(\mathrm{IC}_{50}\right.$ was the concentration which resulted in 50\% inhibition and was determined by XLFit software, equation 205. Inhibition index was the sum of inhibition rates at each tested drug concentration). AsPC-1, BxPC-3, MIA PaCa-2, PANC-1, Capan-1 cell lines were tested against gemcitabine according to the same protocol.

In vitro 3-Dimension colony formation assay. The 96-well tissue culture plates were coated with $50 \mu \mathrm{l}$ of $0.6 \%$ agarose (Takara, Japan) in PBS and then solidified at $4^{\circ} \mathrm{C}$ overnight. Tumor cells which grew as a 2-D monolayer were trypsinized and seeded into each well (2,000 cells in $100 \mu 1$ medium-matrigel mixture). Plates were incubated at $37^{\circ} \mathrm{C}$ overnight and $100 \mu \mathrm{l}$ fresh culture medium was added into each well the next day. After a 6-day
Table I. STR profile of the two pancreatic cancer cell lines.

\begin{tabular}{lcc}
\hline STR locus & PAXC-002 & PAXC-003 \\
\hline Amelogenin & $\mathrm{x}$ & $\mathrm{x}, \mathrm{y}$ \\
THO1 & 7,9 & 9 \\
TPOX & 8,11 & 8,11 \\
D13S317 & 11,12 & 10 \\
vWA & 16,18 & 16 \\
D16S539 & 9 & 12,13 \\
D5S818 & 12 & 12,13 \\
CSF1PO & 12 & 10,12 \\
D7S820 & 8,9 & 11,12
\end{tabular}

incubation, the colony formation were counted under the microscope and measured by Alamar Blue method (Invitrogen).

Cancer stem cell marker expression detection. Cells were harvested, washed in pre-cooled serum-free 1640 medium and then stained with PE conjugated anti-CD133 antibody (Miltenyi Biotec, Germany) on ice for $1 \mathrm{~h}$ and washed three times with PBS. Flow cytometry was done using a BD FACSCalibur flow cytometer.

Statistical analysis. Data were expressed as the mean \pm SD and the in vivo tumor growth was expressed as the mean \pm SEM. Statistically significant differences were determined by the Student's t-test, where appropriate, and defined as $\mathrm{P}<0.05$.

\section{Results}

Establishment of PDA cell lines and characterization. Clinical resected pancreatic tumor samples were subcutaneously implanted into the SCID mice, and tumors developed successfully after about two months. The human primary tumors were removed and placed in culture as described in Materials and methods. After several weeks of cultivation, outgrowth of both epithelioid cells and fibroblast-like cells were observed from the explanted pieces of tumor tissue. To separate the presumed epithelioid tumor cells from the fibroblast-like cells, controlled trypsinizations were optimized and adopted. The cells were digested and split into new tissue culture flasks when they grew to $\sim 80 \%$ confluence. After several passages in culture, the contaminated fibroblasts were removed completely. These tumor cells could be subcultured continuously in vitro ( $>50$ passages in our lab) and were designated as PAXC-002 and PAXC-003, respectively. Both cell lines were free of mycoplasma contamination and deposited at China Center for Typical Culture Collection for public research (CCTCC, the deposit number of PAXC-002 is C201007 and PAXC-003's no. is C201010).

The tumor cell lines were banked at the low passages in liquid nitrogen tanks and characterized after passage 20 when the growth kinetics became stable. Both the cell lines grew with typical slabstone-like epithelioid morphology on plastic surfaces (Fig. 1). The PAXC-002 cells showed obvious clonal shape while the PAXC-003 cells grew more evenly. 


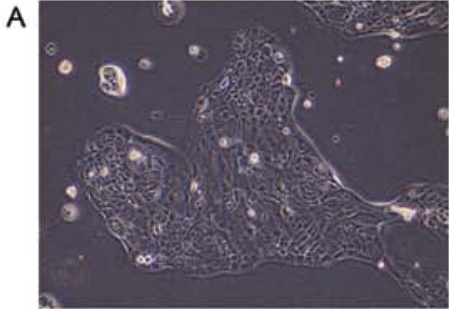

PAXC-002 cell line

B
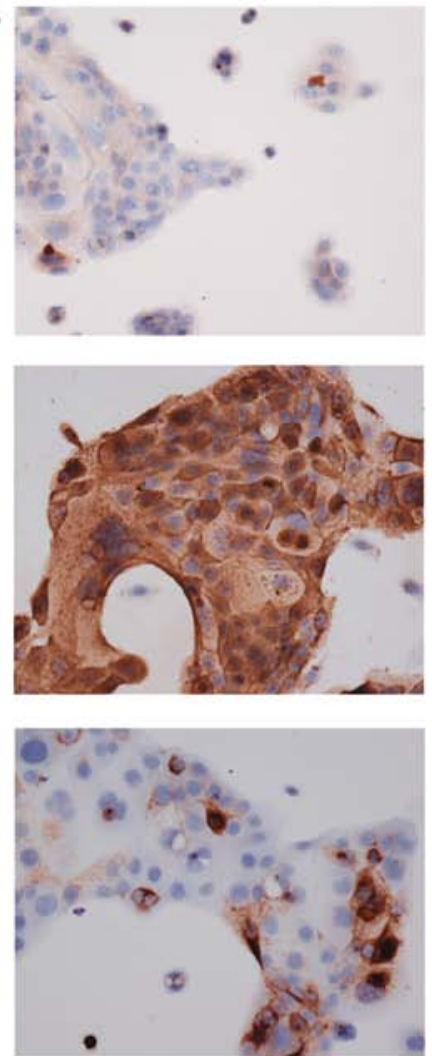

PAXC-002 cell line

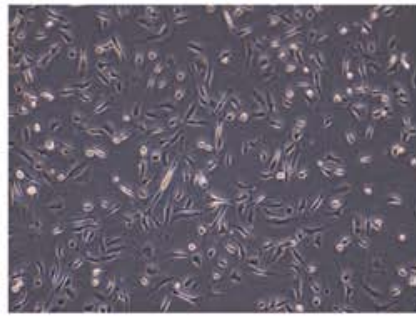

PAXC-003 cell line
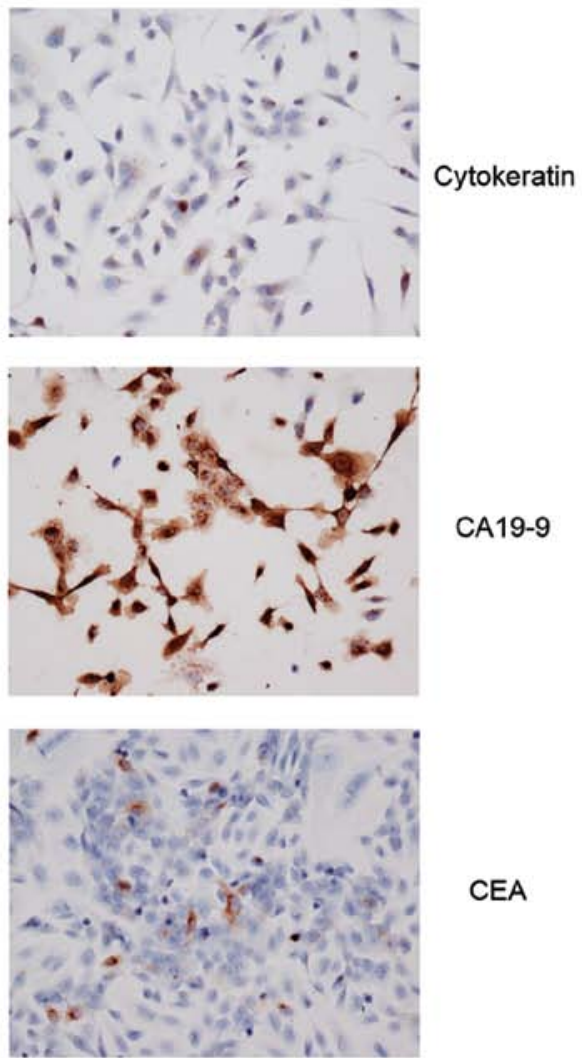

PAXC-003 cell line

C

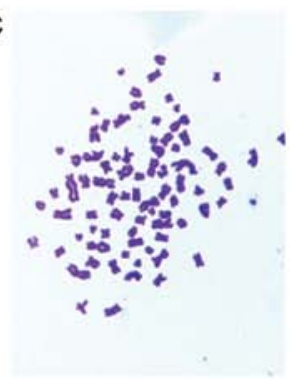

PAXC-002 cell line

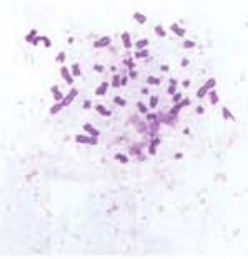

PAXC-003 cell line

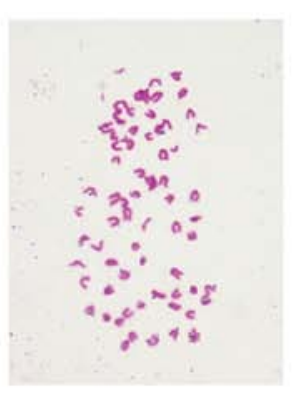

Mouse cells

Figure 1. Morphological studies of the novel pancreatic cancer cell lines. (A) PAXC-002 and PAXC-003 cell lines were observed under an inverted microscope. Magnification x100. (B) Identification of pancreatic cancer markers, including cytokeratin, CA19-9 and CEA. Tumor cells growing on chamber slide were fixed and stained with antibody against cytokeratin, CA19-9 and CEA. Antigen presences were visualized by DAB methods. Magnification x200. (C) Chromosome analysis of PAXC-002, PAXC-003 and mouse cells. Human cell chromosomes had median centromere, but the mouse cells had telocentric chromosome. Magnification x1000.

In order to confirm the human origin and identity of these cell lines, studies were made of chromosome and STR analysis, and immunocytochemistry staining. The results demonstrated that both cell lines had obvious median centromere in all chromosomes to indicate their human cell origin (25) distinguished from the mouse telocentric chromosome (26) (Fig. 1).
PAXC-002 cells had a modal chromosome number of $80 \pm 4$, and the modal number of PAXC- 003 was $43 \pm 3$. The aberrant chromosome number suggested the malignant phenotype of these cells. In addition, the STR profiling of each cell line were tested to avoid the possibility of cell line cross-contamination $(27,28)$. The data of the 8 core loci (Table I) were searched 

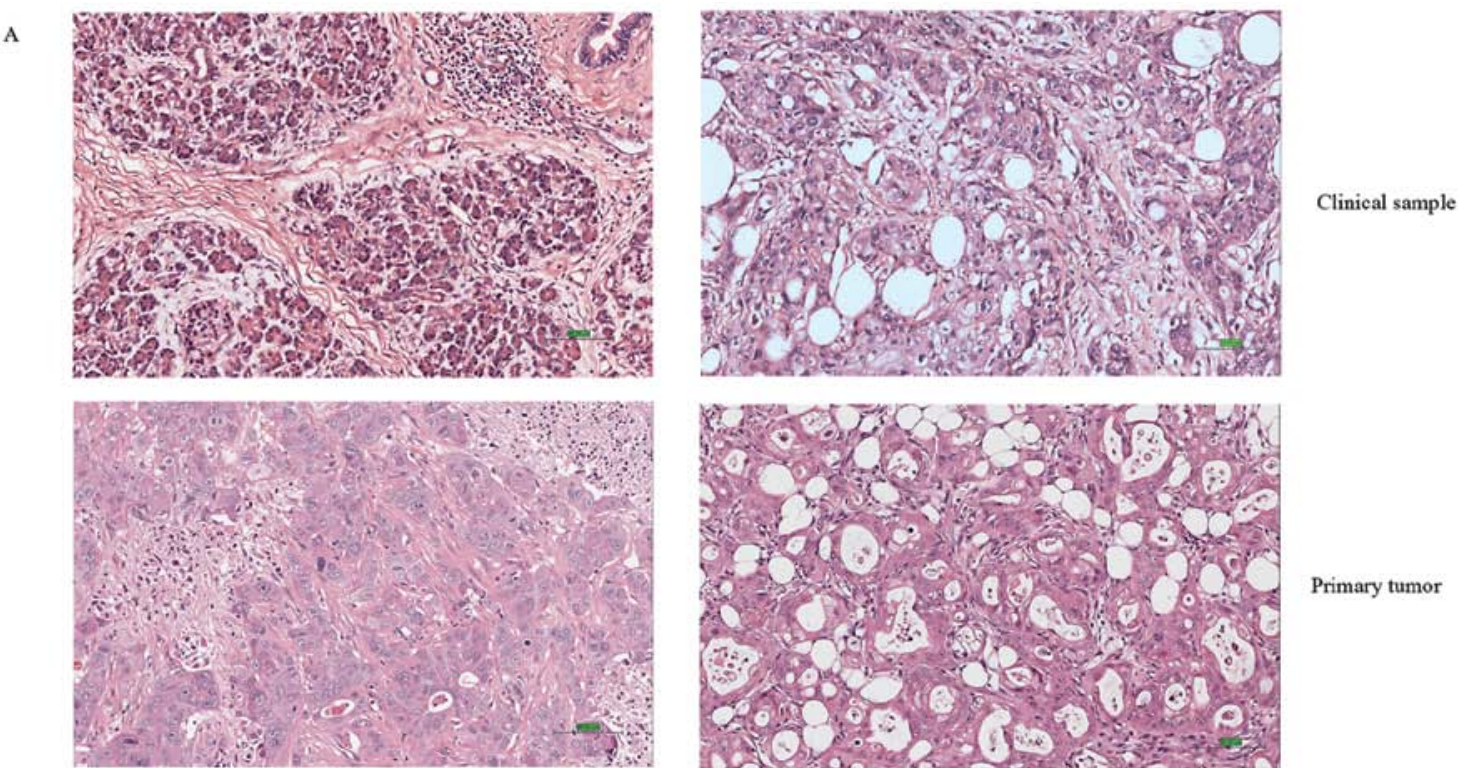

Primary tumo

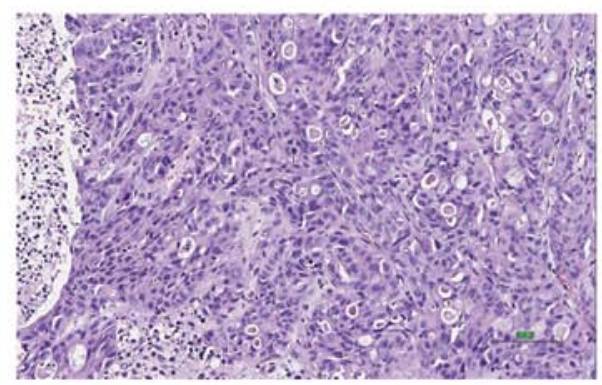

PAXC-002

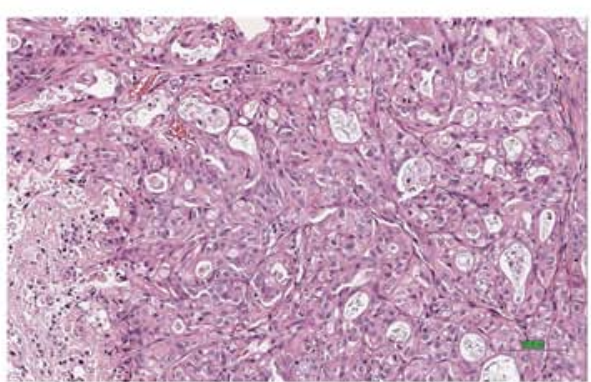

Cell line xenograft

B

Tumor growth of pancreatic cancer cell lines

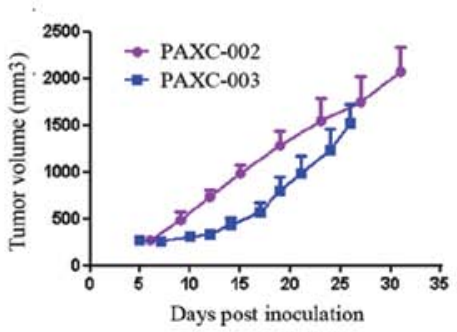

Figure 2. In vivo tumorigenicity test of the pancreatic cancer cell lines. (A) H\&E staining of the clinical samples, primary tumors generated in mice and the cell line xenograft tumors of the pancreatic cancer cell lines. Magnification x200. (B) Tumor growth were monitored twice a week and the tumor volume was measured by a digital caliper.

in ATCC and DSMZ STR databank and did not return any similar results. Furthermore, as seen in Fig. 1, these cell lines are positive to the pancreatic cancer markers, cytokeratin, CA19-9 and carcinoembryonic antigen (CEA). All these data indicated they were novel human pancreatic cancer cell lines.

PAXC-002 cells were routinely subcultured at a ratio of 1:2-1:3 twice a week with a population doubling time of $\sim 48 \mathrm{~h}$ in $\log$ growth phase. PAXC-003 cells could be split at a ratio of 1:3-1:4 twice a week with a population doubling time of $\sim 34 \mathrm{~h}$.

In vivo tumorigenicity test and pathological diagnosis. PAXC002 and PAXC-003 pancreatic cancer cells were harvested and injected into the SCID mice subcutaneously with matrigel.
Tumors developed successfully in all mice. When the tumors reached $\sim 1,500 \mathrm{~mm}^{3}$, the mice were euthanized and the tumor tissue was fixed, paraffin embedded and pathologically diagnosed. As shown in Fig. 2, the H\&E staining of the cell line xenograft tumors were compared with their parent clinical tumor samples and the corresponding human primary tumors generated in mice, and they preserved similar histological characteristics and differentiation (Fig. 2 and Table II). H\&E staining showed that PAXC-002 tumors were composed of a mixture of densely packed, small irregular glands as well as solid tumor cell sheets and nests, and the tumor cells had large neclei with marked pleomorphism. On the other hand, PAXC003 tumors showed a mixture of medium-sized, incompletely duct-like and tubular structures of variable shapes. In summary, 
Table II. Pathological characterization of the pancreatic tumors.

Pathological diagnosis

Clinical samples

Human primary tumors generated in mice

Cell line xenograft tumors

Pancreas: poorly to moderately differentiated PDA differentiated PDA

\section{PAXC-003}

Uncinate process of the pancreas: moderately differentiated PDA

Pancreas: moderately differentiated PDA

Pancreas: moderately differentiated PDA

When tumors grew to appropriate volume, the viable tumor tissues were fixed, paraffin-embedded, stained with H\&E and diagnosed, as shown in Fig. 2.

Table III. In vitro growth inhibition of gemcitabine on pancreatic tumors.

\begin{tabular}{lrc}
\hline Cell line & $\mathrm{IC}_{50}(\mathrm{nM})$ & Inhibition index \\
\hline PAXC-002 & $>100000$ & 99.006 \\
PAXC-003 & 14.564 & 517.114 \\
AsPC-1 & 568.354 & 324.102 \\
BxPC-3 & 13.683 & 525.234 \\
Capan-1 & 6.614 & 462.709 \\
MIA PaCa-2 & 7.734 & 545.304 \\
PANC-1 & 136.786 & 324.602 \\
\hline
\end{tabular}

$\mathrm{IC}_{50}$ was the concentration which resulted in $50 \%$ inhibition and inhibition index is the sum of inhibition rates at each test drug concentration (Fig. 3).

PAXC-002 is a poorly to moderately differentiated pancreatic ductal adenocarcinoma (PDA) cell line, and PAXC-003 is a moderately differentiated PDA cell line.

In vitro growth inhibition assays. Gemcitabine is the standardof-care chemotherapy of pancreatic cancer and it is very potent in in vitro growth inhibition assays with $\mathrm{IC}_{50}$ s between 2 and $20 \mathrm{nM}$ on most pancreatic cancer cell lines $(29,30)$. Exponentially growing PAXC-002, PAXC-003, AsPC-1, BxPC-3, MIA PaCa-2, PANC-1 and Capan-1 cells were exposed to serially diluted gemcitabine for $96 \mathrm{~h}$ and the cell viability was measured by using CellTiter Glo method and the $\mathrm{IC}_{50}$ of gemcitabine in each cell line were determined by XLFit software. PAXC-003, BxPC-3, MIA PaCa-2, PANC-1 and Capan-1 cells were very sensitive to gemcitabine which $\mathrm{IC}_{50} \mathrm{~s}$ ranged from 1 to $20 \mathrm{nM}$. AsPC-1 cell line moderately tolerated gemcitabine with an $\mathrm{IC}_{50}$ concentration of $568 \mathrm{nM}$. However, the $\mathrm{IC}_{50}$ of PAXC-002 cell line was more than $100 \mu \mathrm{M}$ and the PAXC-002 cell viability remains $>50 \%$ even at $100 \mu \mathrm{M}$ of gemcitabine, which meant this cell line was intrinsically resistant to gemcitabine (the results are summarized in Fig. 3 and Table III).

In vitro 3-D colony formation assay. The in vitro 3-D culture model mimics the in vivo physiological property. After a 6-day
Gemcitabine on pancreatic cancer cell lines
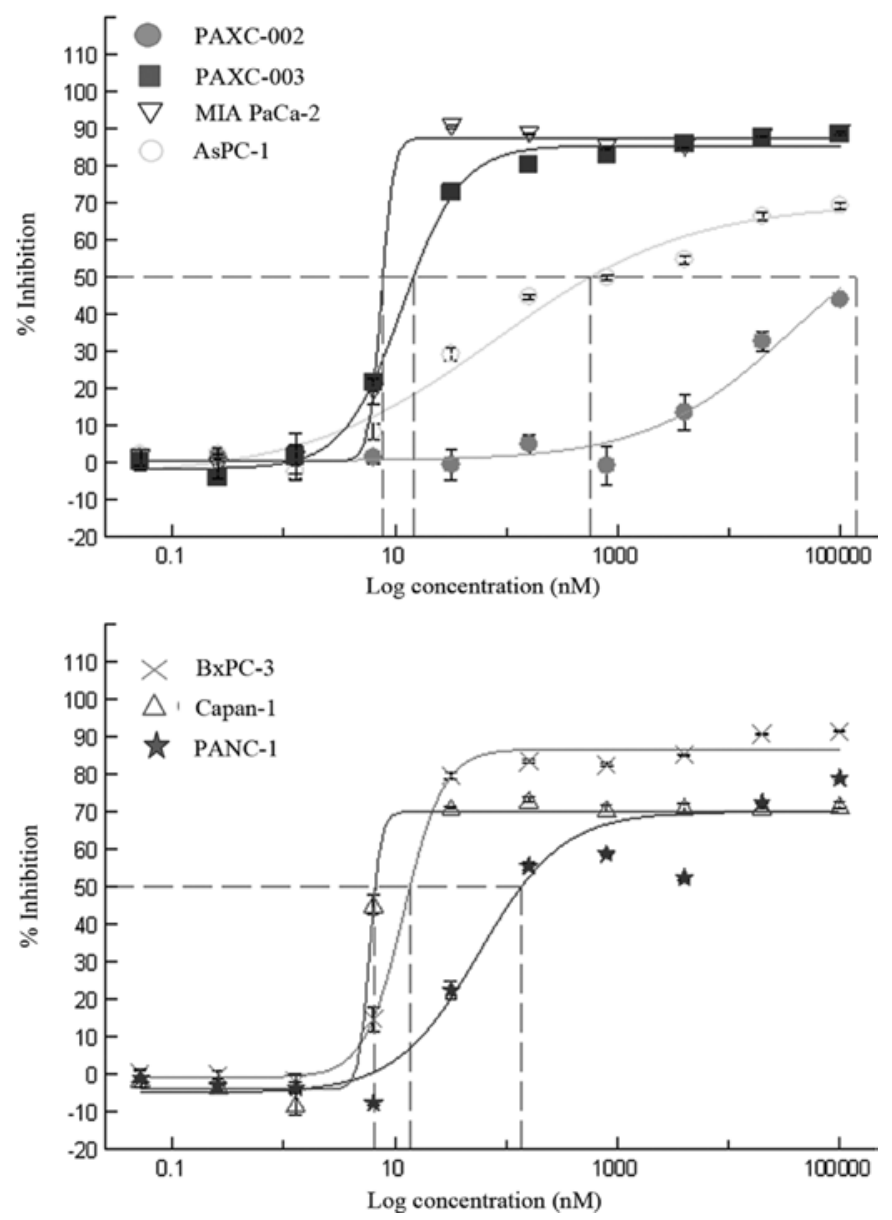

Figure 3. The in vitro anti-proliferation test of gemcitabine on pancreatic cancer cell lines. Gemcitabine concentration was started from $100 \mu \mathrm{M}$ and 1:5 serial diluted (10 points). After 96-h drug exposure, cell viabilities were measured by CellTiter Glo methods and $\mathrm{IC}_{50}$ were determined by XLFit software.

incubation, both cancer cell lines formed colonies in matrix. The gemcitabine-resistant PAXC-002 colonies were significantly more abundant than drug-sensitive PAXC-003 line (Fig. 4). In addition, the colony viability measured by Alamar blue method confirmed the increased colony formation ability of PAXC-002 cells (Fig. 4). This result demonstrated that gemcitabine-resistant 
A

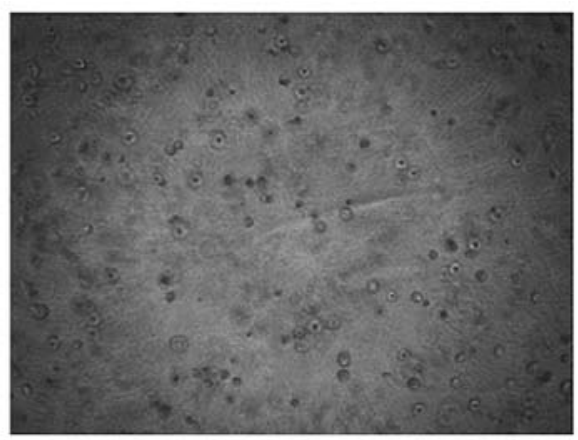

PAXC-002

B

3-D culture of pancreatic cancer cell lines Alamar blue assay

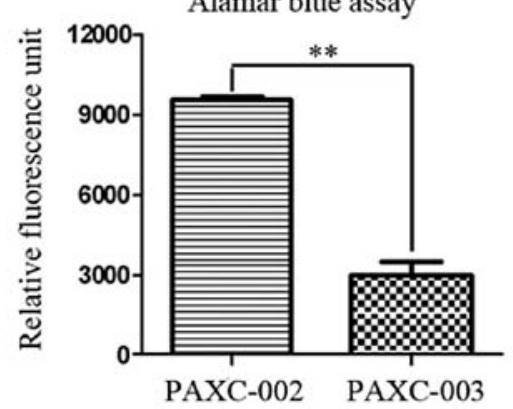

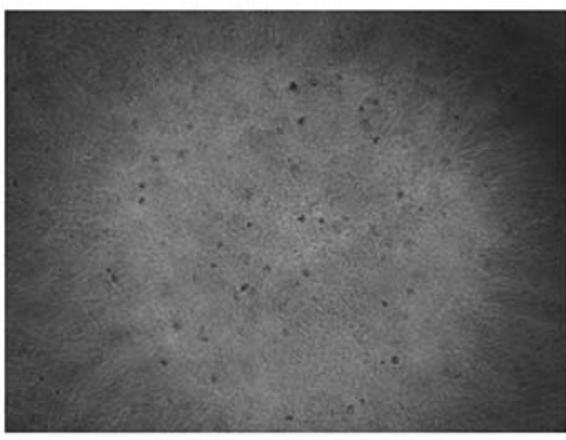

PAXC-003

C 3-D culture of pancreatic cancer cell lines

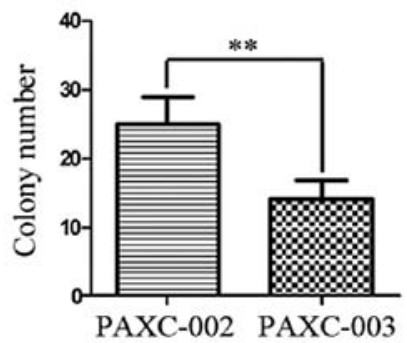

Figure 4. In vitro 3-D colony formation assay. (A) PAXC-002 and PAXC-003 cells were seeded in matrigel and grew in 3-D culture conditions. Magnification x40. (B) Colony viabilities were measured by Alamar blue method. (C) Tumor cell colonies were counted under an inverted microscope. ${ }^{* *} \mathrm{P}<0.001$.

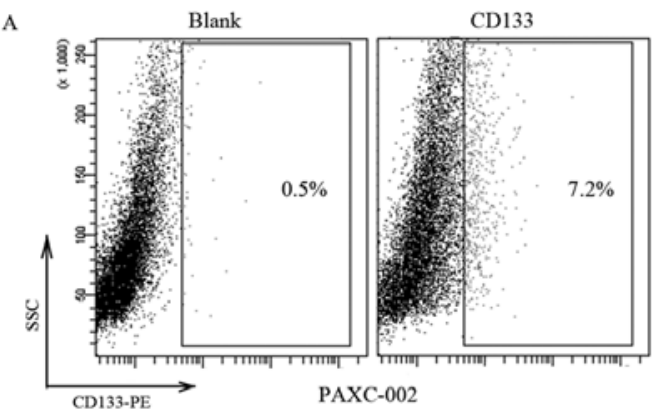

B

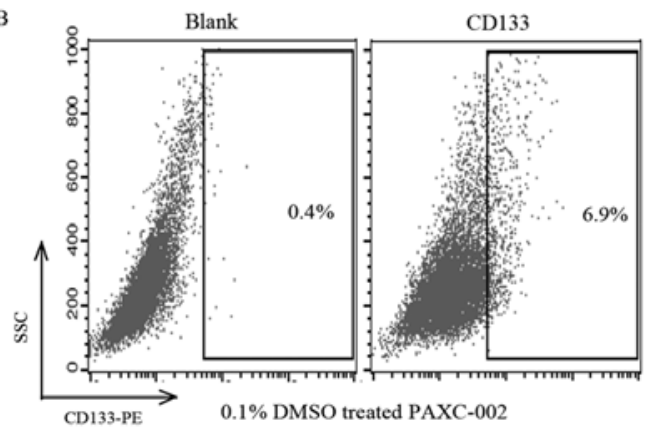

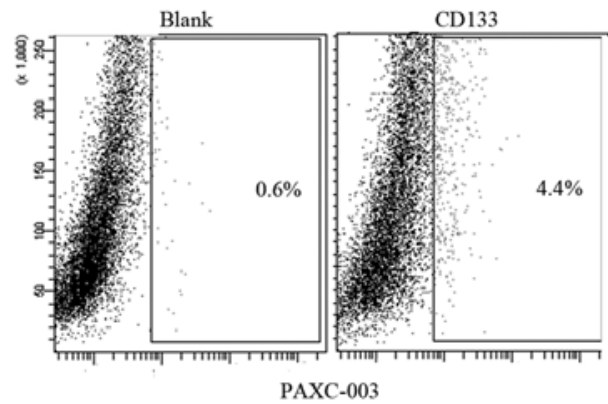

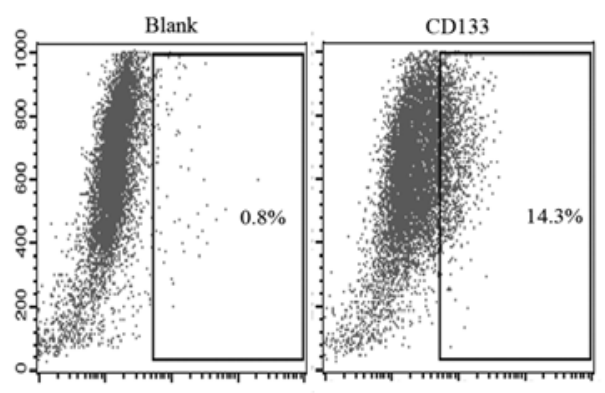

$20 \mu \mathrm{M}$ gemcitabine treated PAXC-002
CD133 positive cells of pancreatic cell lines

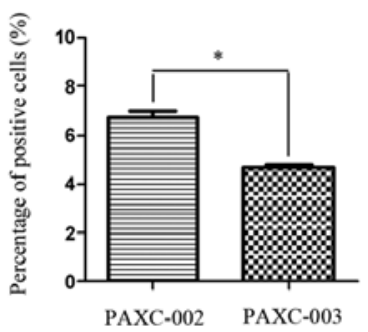

CD133 positive cells of PAXC-002 cells

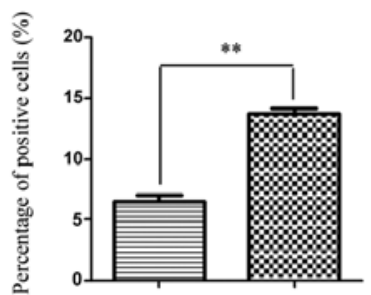

DMSO control $20 \mu \mathrm{M}$ gemeitabine

Figure 5. CD133 was used as the cancer stem cell marker in pancreatic cancer cells. (A) PAXC-002 and PAXC-003 were stained with CD133-PE antibody. (B) PAXC-002 cells were treated with $0.1 \%$ DMSO or $20 \mu \mathrm{M}$ gemcitabine, and then the viable cells were stained with CD133-PE. Plots are representative examples of CD133 staining from three individual experiments, with the frequency of the CD133 positive population as a percentage of cancer cells in the specimen. ${ }^{*} \mathrm{P}<0.05 ;{ }^{* *} \mathrm{P}<0.001$.

PAXC-002 cell line had more in vitro tumorigenic capability indicating the cancer stem cell phenotype.
Cancer stem cell marker expression. The expression of CD133, a cancer stem cell marker which had been reported in pancreatic 
carcinoma $(18,31)$, was examined in these two cancer cell lines. As demonstrated in Fig. 5, there were $6.7 \pm 0.2 \%$ cells positive of CD133 in the gemcitabine-resistant PAXC-002 cell population, whereas only $4.7 \pm 0.1 \%$ cells of drug sensitive PAXC-003 were CD133 positive $(\mathrm{P}<0.05)$. This suggested there were an increased percentage of cancer stem-like cells in gemcitabine-resistant PAXC-002 cell line.

Gemcitabine could only inhibit $\sim 50 \%$ of PAXC-002 cell growth even at $100 \mu \mathrm{M}$ after a 96-h exposure (Fig. 4) and the CD133 expression were measured between the vehicle control (0.1\% DMSO) and gemcitabine treated PAXC-002 cells. The percentage of CD133 positive PAXC-002 cells was elevated after $20 \mu \mathrm{M}$ gemcitabine treatment (6.5 vs. 13.8\%, Fig. 5), which suggested the correlation of drug resistance and CD133 positive stem-like phenotype.

\section{Discussion}

The present report describes the establishment and characterization of the novel pancreatic cancer cell lines PAXC-002 and PAXC-003, derived from human pancreatic ductal adenocarcinomas. It was found that PAXC-002 cells were intrinsically resistant to gemcitabine. Interestingly, the drug resistant cells had more cancer stem cell-like property, which indicated the correlation of gemcitabine resistance and cancer stem cell phenotype.

In this study, both cell lines were established from the in vivo passaged human pancreatic cancer xenografts, which facilitated the human tumor cell growth in vitro. Although there was the possibility of mouse fibroblast contamination in the primary culture, the mouse cells were eliminated by control trypsinizations after several passages (32), and the purity of human cell origin was confirmed by their epithelioid morphology and median centromere-chromosome. The STR profile also showed that they were novel cell lines and free from cell line cross-contamination. The expression of cytokeratin, CA19-9 and CEA (33-35), and tumorigenicity in mice indicated that they were pancreatic cancer cells. Moreover, the corresponding cell line xenograft tumors preserved similar pathological profiles and differentiation compared with their clinical samples and human primary tumors, which suggested that these low passage tumor cells maintained the clinical characteristics of pancreatic carcinoma, which could represent pancreatic tumor models both in vitro and in vivo, and help in translation of clinical information.

Gemcitabine has become the first-line chemotherapy of pancreatic cancer prolonging patient survival and improving the quality of life. However, patients usually have limited response to this therapy, even in combinations, mainly due to the drug resistance of pancreatic carcinomas. Cellular resistance to gemcitabine can be intrinsic or acquired during long-term drug treatment, which is under wide investigation. In this study, PAXC-002 cell line was found innate resistant to gemcitabine compared with PAXC-003 and other widely used pancreatic cancer cell lines (AsPC-1, BxPC-3, MIA PaCa-2, PANC-1 and Capan-1). Unlike some previous studies $(17,36)$, gemcitabine exerted potent growth inhibition effect on these pancreatic cell lines (the $\mathrm{IC}_{50}$ concentration of these lines mainly ranged from tens to hundreds of nano-molar) in our assay format, and these data emphasized the significance of the intrinsically resistant PAXC-002 cell line, which $\mathrm{IC}_{50}$ was more than $100 \mu \mathrm{M}$. It is of interest to find out how PAXC-002 cells were resistant to gemcitabine.

The limited drug efficacy is due to many different mechanisms, including abnormal membrane receptor transport, inefficient metabolic drug conversion or increased metabolite inactivation, enhanced DNA repair and alterations in the apoptotic pathways, and even the tumor microenvironment (10). Recently, the study of cancer stem cells gave new insight into drug resistance. Cancer stem cells represent only a small fraction of tumors, and they possess the self-renewal capability to regenerate a tumor in vivo and in vitro. They are relative quiescent, resistant to drugs and toxins, tolerate apoptosis and have activated DNA-repair mechanism $(37,38)$. The in vitro 3-D colony formation assay is used to measure the self-renewal ability, in which tumor cells could represent in vivo physiological properties (38). In our study, PAXC-002 cells formed more colonies in the matrix, which indicated the drug resistant PAXC-002 cells had more tumorigenic cells compared with the drug sensitive PAXC-003 cells. Furthermore, previous studies used $\mathrm{CD}_{133}+$, or $\mathrm{CD} 24^{+} / \mathrm{CD} 44^{+} / \mathrm{ESA}^{+}$or $\mathrm{ALDH}$ to identify the pancreatic cancer stem cells $(18,19,39,40)$. Here we used CD133 to identify the cancer stem cells, and the CD133positive stem-like cells were more abundant in drug-resistant PAXC-002 cells than that in the sensitive PAXC-003 cells. Furthermore, the CD133 positive PAXC-002 cells increased markedly after gemcitabine treatment. It suggested that the CD133 positive cells could survive the drug exposure, or the drug treatment would stimulate CD133 expression and stem cell phenotype. All these results indicated that CD133-positive stem-like cells would be responsible for the resistance.

In conclusion, we established the novel human pancreatic carcinoma cell lines PAXC-002 and PAXC-003, and PAXC002 is intrinsically resistant to gemcitabine. Furthermore, we find its drug resistance may associate with cancer stem cell-like phenotype. The molecular mechanisms of the drug resistance need to be further investigated. Moreover, the novel cell lines of PAXC-002 and PAXC-003 could be useful in cancer research and anticancer drug screening.

\section{Acknowledgements}

This work was supported by 2010 Yangtze River Delta region cooperation grant (no. 10495810900), Mega-projects of Science Research for the 11th Five-Year Plan (No. 2009ZX09302-002) and the 111 Project (111-2-07).

\section{References}

1. China Health Statistical Yearbook. Ministry of Health, China, 2010. http://www.moh.gov.cn/publicfiles/business/htmlfiles/ zwgkzt/ptjnj/year2010/index2010.html

2. CasperES, Green MR,Kelsen DP, et al: Phase II trial of gemcitabine (2,2'-difluorodeoxycytidine) in patients with adenocarcinoma of the pancreas. Invest New Drugs 12: 29-34, 1994.

3. Rothenberg ML, Moore MJ, Cripps MC, et al: A phase II trial of gemcitabine in patients with 5-FU-refractory pancreas cancer. Ann Oncol 7: 347-353, 1996.

4. Burris HA III, Moore MJ, Andersen J, et al: Improvements in survival and clinical benefit with gemcitabine as first-line therapy for patients with advanced pancreas cancer: a randomized trial. J Clin Oncol 15: 2403-2413, 1997.

5. Ishii H,Furuse J, Nagase M and Yoshino M: Impact of gemcitabine on the treatment of metastatic pancreatic cancer. J Gastroenterol Hepatol 20: 62-66, 2005. 
6. Moore MJ, Goldstein D, Hamm J, et al: Erlotinib plus gemcitabine compared with gemcitabine alone in patients with advanced pancreatic cancer: a phase III trial of the National Cancer Institute of Canada Clinical Trials Group. J Clin Oncol 25: 1960-1966, 2007.

7. Louvet C, Labianca R, Hammel P, et al: Gemcitabine in combination with oxaliplatin compared with gemcitabine alone in locally advanced or metastatic pancreatic cancer: results of a GERCOR and GISCAD phase III trial. J Clin Oncol 23: 3509-3516, 2005.

8. Xiong HQ, Rosenberg A, Lo Buglio A, et al: Cetuximab, a monoclonal antibody targeting the epidermal growth factor receptor in combination with gemcitabine for advanced pancreatic cancer: a multicenter phase II Trial. J Clin Oncol 22: 2610-2616, 2004.

9. Herrmann R, Bodoky G, Ruhstaller T, et al: Gemcitabine plus capecitabine compared with gemcitabine alone in advanced pancreatic cancer: a randomized, multicenter, phase III trial of the Swiss Group for Clinical Cancer Research and the Central European Cooperative Oncology Group. J Clin Oncol 25 2212-2217, 2007

10. El Maalouf G, Le Tourneau C, Batty GN, Faivre S and Raymond E: Markers involved in resistance to cytotoxics and targeted therapeutics in pancreatic cancer. Cancer Treat Rev 35 167-174, 2009.

11. Mackey JR, Mani RS, Selner M, et al: Functional nucleoside transporters are required for gemcitabine influx and manifestation of toxicity in cancer cell lines. Cancer Res 58: 4349-4357, 1998.

12. Spratlin J, Sangha R, Glubrecht D, et al: The absence of human equilibrative nucleoside transporter 1 is associated with reduced survival in patients with gemcitabine-treated pancreas adenocarcinoma. Clin Cancer Res 10: 6956-6961, 2004.

13. Giovannetti E, Del Tacca M, Mey V, et al: Transcription analysis of human equilibrative nucleoside transporter-1 predicts survival in pancreas cancer patients treated with gemcitabine. Cancer Res 66: 3928-3935, 2006.

14. Goan YG, Zhou B, Hu E, Mi S and Yen Y: Overexpression of ribonucleotide reductase as a mechanism of resistance to 2 , 2 -difluorodeoxycytidine in the human KB cancer cell line. Cancer Res 59: 4204-4207, 1999.

15. Graber HU, Friess H, Zimmermann A, et al: Bak expression and cell death occur in peritumorous tissue but not in pancreatic cancer cells. J Gastrointest Surg 3: 74-81, 1999.

16. Noma B, Sasaki T, Fujimoto Y, et al: Expression of multidrug resistance-associated protein 2 is involved in chemotherapy resistance in human pancreatic cancer. Int J Oncol 33: 1187 1194,2008

17. Huanwen W, Zhiyong L, Xiaohua S, Xinyu R, Kai W and Tonghua L: Intrinsic chemoresistance to gemcitabine is associated with constitutive and laminin-induced phosphorylation of FAK in pancreatic cancer cell lines. Mol Cancer 8: 125, 2009.

18. Hermann PC, Huber SL, Herrler T, et al: Distinct populations of cancer stem cells determine tumor growth and metastatic activity in human pancreatic cancer. Cell Stem Cell 1: 313-323, 2007.

19. Li C, Heidt DG, Dalerba P, et al: Identification of pancreatic cancer stem cells. Cancer Res 67: 1030-1037, 2007.

20. Shah AN, Summy JM, Zhang J, Park SI, Parikh NU and Gallick GE: Development and characterization of gemcitabineresistant pancreatic tumor cells. Ann Surg Oncol 14: 3629-3637, 2007.
21. Du Z, Qin R, Wei C, et al: Pancreatic cancer cells resistant to chemoradiotherapy rich in 'stem-cell-like' tumor cells. Dig Dis Sci 56: 741-750, 2011.

22. Yauch RL, Gould SE, Scales SJ, et al: A paracrine requirement for hedgehog signalling in cancer. Nature 455: 406-410, 2008.

23. Tian H, Callahan CA, DuPree KJ, et al: Hedgehog signaling is restricted to the stromal compartment during pancreatic carcinogenesis. Proc Natl Acad Sci USA 106: 4254-4259, 2009.

24. Olive KP, Jacobetz MA, Davidson CJ, et al: Inhibition of Hedgehog signaling enhances delivery of chemotherapy in a mouse model of pancreatic cancer. Science 324: 1457-1461, 2009.

25. Levan A, Fredga K and Sandberg AA: Nomenclature for centromeric position on chromosomes. Hereditas 52: 201-220, 1964

26. Weiss MC and Green H: Human-mouse hybrid cell lines containing partial complements of human chromosomes and functioning human genes. Proc Natl Acad Sci USA 58: 1104-1111, 1967.

27. Masters JR, Thomson JA, Daly-Burns B, et al: Short tandem repeat profiling provides an international reference standard for human cell lines. Proc Natl Acad Sci USA 98: 8012-8017, 2001.

28. Cabrera CM, Cobo F, Nieto A, et al: Identity tests: determination of cell line cross-contamination. Cytotechnology 51: 45-50, 2006.

29. Takano S, Togawa A, Yoshitomi H, et al: Annexin II overexpression predicts rapid recurrence after surgery in pancreatic cancer patients undergoing gemcitabine-adjuvant chemotherapy. Ann Surg Oncol 15: 3157-3168, 2008

30. Giovannetti E, Mey V, Danesi R, Mosca I and Del Tacca M: Synergistic cytotoxicity and pharmacogenetics of gemcitabine and pemetrexed combination in pancreatic cancer cell lines. Clin Cancer Res 10: 2936-2943, 2004

31. Dembinski JL and Krauss S: Characterization and functional analysis of a slow cycling stem cell-like subpopulation in pancreas adenocarcinoma. Clin Exp Metastasis 26: 611-623, 2009.

32. Dangles-Marie V, Pocard M, Richon S, et al: Establishment of human colon cancer cell lines from fresh tumors versus xenografts: comparison of success rate and cell line features. Cancer Res 67: 398-407, 2007.

33. Haglund C, Roberts PJ, Kuusela P, Scheinin TM, Makela O and Jalanko H: Evaluation of CA 19-9 as a serum tumour marker in pancreatic cancer. Br J Cancer 53: 197-202, 1986.

34. Iwamura T, Taniguchi S, Kitamura N, et al: Correlation between CA19-9 production in vitro and histological grades of differentiation in vivo in clones isolated from a human pancreatic cancer cell line (SUIT-2). J Gastroenterol Hepatol 7: 512-519, 1992.

35. Lundin J, Roberts PJ, Kuusela P and Haglund C: The prognostic value of preoperative serum levels of CA 19-9 and CEA in patients with pancreatic cancer. Br J Cancer 69: 515-519, 1994.

36. Arumugam T, Ramachandran V, Fournier KF, et al: Epithelial to mesenchymal transition contributes to drug resistance in pancreatic cancer. Cancer Res 69: 5820-5828, 2009.

37. Dean M, Fojo T and Bates S: Tumour stem cells and drug resistance. Nat Rev Cancer 5: 275-284, 2005.

38. Clarke MF, Dick JE, Dirks PB, et al: Cancer stem cells-perspectives on current status and future directions: AACR workshop on cancer stem cells. Cancer Res 66: 9339-9344, 2006.

39. Lee CJ, Dosch J and Simeone DM: Pancreatic cancer stem cells. J Clin Oncol 26: 2806-2812, 2008

40. Jimeno A, Feldmann G, Suárez-Gauthier A, et al: A direct pancreatic cancer xenograft model as a platform for cancer stem cell therapeutic development. Mol Cancer Ther 8: 310-314, 2009. 